\title{
Fuzzy and anti fuzzy ternary hypergroups
}

\author{
Bijan Davvaz \\ Department of Mathematics, Yazd University, Yazd, Iran \\ davvaz@yazduni.ac.ir, bdavvaz@yahoo.com
}

\begin{abstract}
The notion of ternary hypergroup is a generalization of the notion of hypergroup in the sense of Marty. In this paper, we study the concepts of fuzzy and anti fuzzy ternary subhypergroups of a ternary hypergroup and we discuss some properties of them.
\end{abstract}

Keywords: Fuzzy subset, Subhypergroup, Ternary hypergroup.

\section{Introduction}

The concept of a fuzzy subset of a non-empty set first was introduced in (Zadeh, 1965). Rosenfeld applied this concept to the theory of groups and defined the concept of a fuzzy subgroup of a group (Rosenfeld, 1971). Since then the study of fuzzy algebraic structures has been pursued in many directions such as groups, rings, vector spaces and fields. The concept of anti fuzzy subgroup was given in (Biswas, 1990). Davvaz applied fuzzy sets to the theory of algebraic hyperstructures (Davvaz, 1999) and defined the concept of fuzzy subhypergroup (respectively, fuzzy $H_{v}$-subgroup).

The concept of $n$-ary hypergroup is defined by Davvaz \& Vougiouklis (2006), which is a generalization of the concept of hypergroup in the sense of Marty and a generalization of $n$-ary group, too. Davvaz and Corsini (2007) introduced the notion of a fuzzy $n$-ary subhypergroup of an $n$-ary hypergroup. Then this concept was further studied by others (Davvaz et al., 2009, 2010; Kazanci et al., 2010, 2011).

A ternary hypergroup is a particular case of an $n$-ary hypergroup for $n=3$. Davvaz and Leoreanu-Fotea (2010) studied the ternary hypergroups associated with binary relations. Davvaz et al. (2011) provided examples of ternary hyperstructures associated with chain reactions in chemistry (Davvaz, 2009). In this paper, we study the concepts of fuzzy and anti fuzzy ternary subhypergroups of a ternary hypergroup and we discuss some properties of them.

\section{Ternary hypergroups}

Let $H$ be a non-empty set and $f: H \times H \times H \rightarrow \wp^{*}(H)$, where $\wp^{*}(H)$ is the set of all non-empty subsets of $H$. Then $f$ is called a ternary hyperoperation on $H$ and the pair $(H, f)$ is called a ternary hypergroupoid. If $A, B, C$ are non-empty subsets of $H$, then we define

$$
f(A, B, C)=\bigcup_{a \in A, b \in B, c \in C} f(a, b, c) \text {. }
$$

Definition 1: The ternary hypergroupoid $(H, f)$ is called a ternary semihypergroup if for every $a_{1}, \ldots, a_{5} \in H$, we have

$f\left(f\left(a_{1}, a_{2}, a_{3}\right), a_{4}, a_{5}\right)=f\left(a_{1}, f\left(a_{2}, a_{3}, a_{4}\right), a_{5}\right)=f\left(a_{1}, a_{2}, f\left(a_{3}, a_{4}, a_{5}\right)\right)$

Definition 2: A ternary semihypergroup $(H, f)$ is called

a ternary hypergroup if for all $a, b, c \in H$ there exist $x, y, z \in H$ such that:

$$
c \in f(x, a, b) \cap f(a, y, b) \cap f(a, b, z) .
$$

Notice that a ternary semigroup $(S, f)$ is said to be a ternary group if it satisfies the following property that for all $a, b, c \in S$, there exist unique $x, y, z \in S$ such that

$$
c=f(x, a, b), c=f(a, y, b), c=f(a, b, z) .
$$

Example 1: Let $Z$ be the set of integer numbers with ternary hyperoperation $f$ defined as follows:

$$
f(x, y, z)=\left\{n_{1} x+n_{2} y+n_{3} z \mid n_{1}, n_{2}, n_{3} \in Z\right\} .
$$

Then $(Z, f)$ is a ternary hypergroup.

Example 2: Let $(L, \vee, \wedge)$ be a modular lattice. For all $a_{1}, a_{2}, a_{3} \in L$, we define $A^{(1)}=a_{2} \vee a_{3}, A^{(2)}=a_{1} \vee a_{3}, A^{(3)}=a_{1} \vee a_{2}$, $A_{3}=a_{1} \vee a_{2} \vee a_{3}$, We define the following ternary hyperoperation on $L$ :

$f\left(a_{1}, a_{2}, a_{3}\right)=\left\{x \in L \mid x \vee A^{(i)}=A_{3}\right.$, for all $\left.i=1,2,3\right\}$.

Notice that the set $f\left(a_{1}, a_{2}, a_{3}\right)$ is not empty, because it contains $x=A_{3}$. Then $(L, f)$ is a ternary hypergroup. Fuzzy ternary subhypergroups

Definition 3: Let $(H, f)$ be a ternary hypergroup and $\mu$ be a fuzzy subset of $H$. Then $\mu$ is said to be a fuzzy ternary subhypergroup of $H$ if the following axioms hold: (1) $\min \{\mu(x), \mu(y), \mu(z)\} \leq \inf _{a \in f(x, y, z)}\{\mu(a)\}$ for all $x, y, z \in H$,

(2) for all $a, b, c \in H$ there exist $x, y, z \in H$ such that $c \in f(x, a, b) \cap f(a, y, b) \cap f(a, b, z)$ and $\min \{\mu(a), \mu(b), \mu(c)\} \leq \min \{\mu(x), \mu(y), \mu(z)\}$.

For any fuzzy subset $\mu$ of a non-empty set $X$ and any 
Indian Journal of Science and Technology

$t \in(0,1]$, we define the set

$$
U(\mu ; t)=\{x \in X \mid \mu(x) \geq t\} .
$$

Theorem 2: Let $(H, f)$ be a ternary hypergroup and $\mu$ be a fuzzy subset of $H$. Then $\mu$ is a fuzzy ternary subhypergroup of $H$ if and only if for every $t \in(0,1]$, $U(\mu ; t)(\neq \varnothing)$ is a ternary subhypergroup of $H$.

Proof. Suppose that $\mu$ is a fuzzy ternary subhypergroup of $H$. For every $x, y, z \in U(\mu ; t)$ we have $\min \{\mu(x), \mu(y), \mu(z)\} \geq t$ and so

$$
\inf _{a \in f(x, y, z)}\{\mu(a)\} \geq t .
$$

Thus, for every $a \in f(x, y, z)$ we have $\mu(a) \geq t$. Therefore, $f(x, y, z) \subseteq U(\mu ; t)$.

Now, if $a, b, c \in U(\mu ; t)$ then there exist $x, y, z \in H$ such that $c \in f(x, a, b) \cap f(a, y, b) \cap f(a, b, z)$ and $\min \{\mu(a), \mu(b), \mu(c)\} \leq \min \{\mu(x), \mu(y), \mu(z)\}$. From $a, b, c \in U(\mu ; t)$, we obtain $\min \{\mu(a), \mu(b), \mu(c)\} \geq t$ and so $\mu(x) \geq t, \mu(y) \geq t, \mu(z) \geq t$. This proves that $x, y, z \in U(\mu ; t)$.

Conversely, assume that for every $0 \leq t \leq 1, U(\mu ; t)$ $(\neq \varnothing)$ is a ternary subhypergroup of $H$. For every $x, y, z \in H$, we put $t_{0}=\min \{\mu(x), \mu(y), \mu(z)\}$. Then $x, y, z \in U\left(\mu ; t_{0}\right)$ and so $f(x, y, z) \subseteq U\left(\mu ; t_{0}\right)$.

Therefore, for every $a \in f(x, y, z)$ we have $\mu(a) \geq t_{0}$ implying that

$$
\min \{\mu(x), \mu(y), \mu(z)\} \leq \inf _{a \in f(x, y, z)}\{\mu(a)\}
$$

and in this way the first condition of Definition 3 is verified. In order to verify the second condition, let $a, b, c \in H$. We put $t_{1}=\min \{\mu(a), \mu(b), \mu(c)\}$. Then, we obtain $a, b, c \in U\left(\mu ; t_{1}\right)$. So , there exist $x, y, z \in U\left(\mu ; t_{1}\right)$ such that $c \in f(x, a, b) \cap f(a, y, b) \cap f(a, b, z)$. On the other hand, since $x, y, z \in U\left(\mu ; t_{1}\right)$, it follows $\mu(x) \geq t_{1}, \mu(y) \geq t_{1}, \mu(z) \geq t_{1}$ and hence

$$
\min \{\mu(a), \mu(b), \mu(c)\} \leq \min \{\mu(x), \mu(y), \mu(z)\} .
$$

Anti fuzzy ternary subhypergroups

Definition 4: Let $(H, f)$ be a ternary hypergroup and $\mu$ be a fuzzy subset of $H$. Then $\mu$ is said to be an anti fuzzy ternary subhypergroup of $H$ if the following axioms hold:

(1) $\sup _{a \in f(x, y, z)}\{\mu(a)\} \leq \max \{\mu(x), \mu(y), \mu(z)\}$ for all $x, y, z \in H$,
Vol. 5 No. 3 (Mar 2012)

ISSN: 0974- 6846
(2) for all $a, b, c \in H$ there exist $x, y, z \in H$ such that $c \in f(x, a, b) \cap f(a, y, b) \cap f(a, b, z)$ and

$$
\max \{\mu(x), \mu(y), \mu(z)\} \leq \max \{\mu(a), \mu(b), \mu(c)\} .
$$

Example 3: Consider the ternary hypergroup $(Z, f)$ defined in Example 1. Let the fuzzy subset $\mu: Z \rightarrow[0,1]$ be as follows:

$$
\mu(x)= \begin{cases}t_{0} & \text { if } x \in<4> \\ t_{1} & \text { if } x \in<2>\backslash<4> \\ t_{2} & \text { if } x \in Z \backslash<2>,\end{cases}
$$

where $t_{0}<t_{1}<t_{2}$. Then $\mu$ is an anti fuzzy ternary subhypergroup.

For any fuzzy subset $\mu$ of a non-empty set $X$ and any $t \in(0,1]$, we define the set

$$
L(\mu ; t)=\{x \in X \mid \mu(x) \leq t\} .
$$

Theorem 2: Let $(H, f)$ be a ternary hypergroup and $\mu$ be a fuzzy subset of $H$. Then $\mu$ is an anti fuzzy ternary subhypergroup of $H$ if and only if for every $t \in(0,1]$, $L(\mu ; t) \quad(\neq \varnothing)$ is a ternary subhypergroup of $H$.

Proof. Suppose that $\mu$ is an anti fuzzy ternary subhypergroup of $H$. For every $x, y, z \in L(\mu ; t)$ we have $\max \{\mu(x), \mu(y), \mu(z)\} \leq t$ and so

$$
\sup _{a \in f(x, y, z)}\{\mu(a)\} \leq t \text {. }
$$

Thus, for every $a \in f(x, y, z)$ we have $\mu(a) \leq t$. Therefore, $f(x, y, z) \subseteq L(\mu ; t)$.

Now, if $a, b, c \in L(\mu ; t)$ then there exist $x, y, z \in H$ such that $c \in f(x, a, b) \cap f(a, y, b) \cap f(a, b, z)$ and $\max \{\mu(x), \mu(y), \mu(z)\} \leq \max \{\mu(a), \mu(b), \mu(c)\}$.

From $a, b, c \in L(\mu ; t)$, we obtain

$$
\max \{\mu(a), \mu(b), \mu(c)\} \leq t
$$

and so $\mu(x) \leq t, \mu(y) \leq t, \mu(z) \leq t$. This proves that $x, y, z \in L(\mu ; t)$.

Conversely, assume that for every $0 \leq t \leq 1$, $L(\mu ; t)(\neq \varnothing)$ is a ternary subhypergroup of $H$. For every $x, y, z \in H$, we put $t_{0}=\max \{\mu(x), \mu(y), \mu(z)\}$. Then $x, y, z \in L\left(\mu ; t_{0}\right)$ and so $f(x, y, z) \subseteq L\left(\mu ; t_{0}\right)$. Therefore, for every $a \in f(x, y, z)$ we have $\mu(a) \leq t_{0}$ implying that

$$
\sup _{a \in f(x, y, z)}\{\mu(a)\} \leq \max \{\mu(x), \mu(y), \mu(z)\}
$$

and in this way the first condition of Definition 4 is verified. In order to verify the second condition, let $a, b, c \in H$. 
We put $t_{1}=\max \{\mu(a), \mu(b), \mu(c)\}$. Then, we have $a, b, c \in L\left(\mu ; t_{1}\right)$. So , there exists $x, y, z \in L\left(\mu ; t_{1}\right)$ such that $c \in f(x, a, b) \cap f(a, y, b) \cap f(a, b, z)$. On the other hand, since $x, y, z \in L\left(\mu ; t_{1}\right)$, it follows

and hence

$$
\mu(x) \leq t_{1}, \mu(y) \leq t_{1}, \mu(z) \leq t_{1}
$$

$$
\max \{\mu(x), \mu(y), \mu(z)\} \leq \max \{\mu(a), \mu(b), \mu(c)\} \text {. }
$$

Theorem 3: Let $(H, f)$ be a ternary hypergroup and $\mu$ be a fuzzy subset of $H$. Then $\mu$ is a fuzzy ternary hypergroup of $H$ if and only if it's complement $\mu^{c}$ is an anti fuzzy ternary hypergroup of $H$.

Proof. Suppose that $\mu$ is a fuzzy ternary hypergroup of $H$. For every $x, y, z$ in $H$, we have

$\min \{\mu(x), \mu(y), \mu(z)\} \leq \inf _{a \in f(x, y, z)}\{\mu(a)\}$, or $\min \left\{1-\mu^{c}(x), 1-\mu^{c}(y), 1-\mu^{c}(z)\right\} \leq \inf _{a \in f(x, y, z)}\left\{1-\mu^{c}(a)\right\}$, or $\min \left\{1-\mu^{c}(x), 1-\mu^{c}(y), 1-\mu^{c}(z)\right\} \leq 1-\sup _{a \in f(x, y, z)}\left\{\mu^{c}(a)\right\}$, or $\sup _{a \in f(x, y, z)}\left\{\mu^{c}(\alpha)\right\} \leq 1-\min \left\{1-\mu^{c}(x), 1-\mu^{c}(y), 1-\mu^{c}(z)\right\}$, or $\sup _{a \in f(x, y, z)}\left\{\mu^{c}(\alpha)\right\} \leq \max \left\{\mu^{c}(x), \mu^{c}(y), \mu^{c}(z)\right\}$, and in this way the condition (1) of Definition 4 is verified for $\mu^{c}$ Since $\mu$ is a fuzzy ternary hypergroup of $H$, so for every $a, b, c \in H$, there exist $x, y, z \in H$ such that $c \in f(x, a, b) \cap f(a, y, b) \cap f(a, b, z)$ and $\min \{\mu(a), \mu(b), \mu(c)\} \leq \min \{\mu(x), \mu(y), \mu(z)\}$ or $\min \left\{1-\mu^{c}(a), 1-\mu^{c}(b), 1-\mu^{c}(c)\right\}$ $\leq \min \left\{1-\mu^{c}(x), 1-\mu^{c}(y), 1-\mu^{c}(z)\right\}$

which implies that $\max \left\{\mu^{c}(x), \mu^{c}(y), \mu^{c}(z)\right\} \leq \max \left\{\mu^{c}(a), \mu^{c}(b), \mu^{c}(c)\right\}$ and the second condition of Definition 4 is satisfied.

Therefore, $\mu^{c}$ is an anti ternary semihypergroup of $H$. The converse also can be proved similarly. Lemma 4: Let $\mu$ be an anti fuzzy ternary subhypergroup of a ternary hypergroup $(H, f)$ and $t_{1}<t_{2}$. Then $L\left(\mu, t_{1}\right)$ and $L\left(\mu, t_{2}\right)$ are equal if there is no $x \in H$ such that $t_{1}<\mu(x) \leq t_{2}$.

Proof. Suppose that $L\left(\mu, t_{1}\right)=L\left(\mu, t_{2}\right)$. If there exists $x \in H$ such that $t_{1}<\mu(x) \leq t_{2}$, then $L\left(\mu, t_{1}\right)$ is a proper subset of $L\left(\mu, t_{2}\right)$ and this is a contradiction.

Conversely, assume that there is no $x \in H$ such that $t_{1}<\mu(x) \leq t_{2}$. Since $t_{1}<t_{2}, L\left(\mu, t_{1}\right) \subseteq L\left(\mu, t_{2}\right)$. Now, let $x \in L\left(\mu, t_{2}\right)$ be an arbitrary element. Then $\mu(x) \leq t_{2}$ and so by hypothesis we conclude that $\mu(x) \leq t_{1}$. Thus, $x \in L\left(\mu, t_{1}\right)$. Therefore, $L\left(\mu, t_{1}\right)=L\left(\mu, t_{2}\right)$.

Proposition 5: Let $(H, f)$ be a ternary hypergroup and $\mu$ be an anti fuzzy ternary subhypergroup of $H$. If Im $(\mu)=\left\{t_{0}, t_{1}, \ldots, t_{n}\right\} \quad$ where $t_{0}<t_{1}<\ldots<t_{n}$, then $L\left(\mu ; t_{i}\right)$ (for $i=1, \ldots, n$ ) constitute all the lower level subsets of $\mu$.

Proof. Suppose that $t \in[0,1]$ and $t \notin \operatorname{Im}(\mu)$. If $t>t_{n}$, then $L\left(\mu, t_{n}\right) \subseteq L(\mu, t)$. Since $L\left(\mu ; t_{n}\right)=H$, it follows that $L(\mu ; t)=H$ and so $L\left(\mu ; t_{n}\right)=L(\mu ; t)$. Now, suppose that $t_{i}<t<t_{i+1}$ where $1 \leq i \leq n-2$. Then there is no $x \in H$ such that $t<\mu(x) \leq t_{i+1}$. By Lemma 4, we have $L(\mu ; t)=L\left(\mu ; t_{i+1}\right)$. This completes the proof.

Proposition 6: Let $(H, f)$ be a ternary hypergroup and $K$ be a non-empty subset of $H$. Let $\mu$ be a fuzzy subset of $H$ such that

$$
\mu(x)= \begin{cases}0 & \text { if } x \in K \\ 1 & \text { if } x \notin K\end{cases}
$$

Then $\mu$ is an anti fuzzy ternary subhypergroup of $H$ if and only if $K$ is a ternary subhypergroup of $H$.

Proof. It is straightforward.

Theorem 7: Let $(H, f)$ be a ternary hypergroup and $K$ be a ternary subhypergroup of $H$. Then for each $t \in[0,1]$, there exists an anti fuzzy ideal $\mu$ of $H$ such that $L(\mu ; t)=K$.

Proof. Suppose that $t \in[0,1]$ and define a fuzzy subset $\mu$ of $H$ by

$$
\mu(x)= \begin{cases}t & \text { if } x \in K \\ 1 & \text { if } x \notin K .\end{cases}
$$

Then, $L(\mu ; t)=K$.

Let $X_{1}, X_{2}$ be two non-empty sets and $\mu, \lambda$ be fuzzy subsets of $X_{1}, X_{2}$, respectively. Then the product of $\mu$ and $\lambda$ is the fuzzy subset $\mu \times \lambda$ of $X_{1} \times X_{2}$ where

$$
(\mu \times \lambda)(x, y)=\min \{\mu(x), \lambda(y)\}
$$

for all $(x, y) \in X_{1} \times X_{2}$.

Theorem 8: Let $\left(H_{1}, f_{1}\right)$ and $\left(H_{2}, f_{2}\right)$ be two ternary hypergroups and $\mu, \lambda$ be anti fuzzy ternary 
subhypergroups of $H_{1}, H_{2}$, respectively. Then $\mu \times \lambda$ is an anti fuzzy ternary subhypergroup of $\mathrm{H}_{1} \times \mathrm{H}_{2}$.

Proof. Let $\left(x_{1}, x_{2}\right),\left(y_{1}, y_{2}\right),\left(z_{1}, z_{2}\right) \in H_{1} \times H_{2}$. For every $\left(a_{1}, a_{2}\right) \in\left(f_{1} \times f_{2}\right)\left(\left(x_{1}, x_{2}\right),\left(y_{1}, y_{2}\right),\left(z_{1}, z_{2}\right)\right)$ we have $(\mu \times \lambda)\left(a_{1}, a_{2}\right)=\min \left\{\mu\left(a_{1}\right), \lambda\left(a_{2}\right)\right\}$

$\leq \min \left\{\max \left\{\mu\left(x_{1}\right), \mu\left(y_{1}\right), \mu\left(z_{1}\right)\right\}, \max \left\{\lambda\left(x_{2}\right), \lambda\left(y_{2}\right), \lambda\left(z_{2}\right)\right\}\right\}$

$=\min \left\{\max \left\{\mu\left(x_{1}\right), \lambda\left(x_{2}\right)\right\}, \max \left\{\mu\left(y_{1}\right), \lambda\left(y_{2}\right)\right\}, \max \left\{\mu\left(z_{1}\right), \lambda\left(z_{2}\right)\right\}\right\}$

$=\max \left\{\min \left\{\mu\left(x_{1}\right), \lambda\left(x_{2}\right)\right\}, \min \left\{\mu\left(y_{1}\right), \lambda\left(y_{2}\right)\right\}, \min \left\{\mu\left(z_{1}\right), \lambda\left(z_{2}\right)\right\}\right\}$

$=\max \left\{(\mu \times \lambda)\left(x_{1}, x_{2}\right),(\mu \times \lambda)\left(y_{1}, y_{2}\right),(\mu \times \lambda)\left(z_{1}, z_{2}\right)\right\}$.

Therefore

$$
\begin{aligned}
& \sup _{\left(a_{1}, a_{2}\right) \in\left(f_{1} \times f_{2}\right)\left(\left(x_{1}, x_{2}\right),\left(y_{1}, y_{2}\right),\left(z_{1}, z_{2}\right)\right)}\left\{(\mu \times \lambda)\left(a_{1}, a_{2}\right)\right\} \\
& \leq \max \left\{(\mu \times \lambda)\left(x_{1}, x_{2}\right),(\mu \times \lambda)\left(y_{1}, y_{2}\right),(\mu \times \lambda)\left(z_{1}, z_{2}\right)\right\} .
\end{aligned}
$$

So, the first condition of Definition 4 is satisfied. Now, we prove the second condition of Definition 4 as follows:

For every $\left(a_{1}, a_{2}\right),\left(b_{1}, b_{2}\right),\left(c_{1}, c_{2}\right) \in H_{1} \times H_{2}$, there exist

$$
\begin{aligned}
& \left(x_{1}, x_{2}\right),\left(y_{1}, y_{2}\right),\left(z_{1}, z_{2}\right) \in H_{1} \times H_{2} \text { with } \\
& \quad c_{1} \in f_{1}\left(x_{1}, a_{1}, b_{1}\right) \cap f_{1}\left(a_{1}, y_{1}, b_{1}\right) \cap f_{1}\left(a_{1}, b_{1}, z_{1}\right)
\end{aligned}
$$

and

$$
c_{2} \in f_{2}\left(x_{2}, a_{2}, b_{2}\right) \cap f_{2}\left(a_{2}, y_{2}, b_{2}\right) \cap f_{2}\left(a_{2}, b_{2}, z_{2}\right)
$$

such that

$$
\max \left\{\mu\left(x_{1}\right), \mu\left(y_{1}\right), \mu\left(z_{1}\right)\right\} \leq \max \left\{\mu\left(a_{1}\right), \mu\left(b_{1}\right), \mu\left(c_{1}\right)\right\} .
$$

and

$\max \left\{\lambda\left(x_{2}\right), \lambda\left(y_{2}\right), \lambda\left(z_{2}\right)\right\} \leq \max \left\{\lambda\left(a_{2}\right), \lambda\left(b_{2}\right), \lambda\left(c_{2}\right)\right\}$. Therefore, we have

$\begin{aligned}\left(c_{1}, c_{2}\right) \in & \left(f_{1} \times f_{2}\right)\left(\left(x_{1}, x_{2}\right),\left(a_{1}, a_{2}\right),\left(b_{1}, b_{2}\right)\right) \cap\left(f_{1} \times f_{2}\right)\left(\left(a_{1}, a_{2}\right),\left(y_{1}, y_{2}\right),\left(b_{1}, b_{2}\right)\right) \\ & \cap\left(f_{1} \times f_{2}\right)\left(\left(a_{1}, a_{2}\right),\left(b_{1}, b_{2}\right),\left(z_{1}, z_{2}\right)\right)\end{aligned}$

$$
\cap\left(f_{1} \times f_{2}\right)\left(\left(a_{1}, a_{2}\right),\left(b_{1}, b_{2}\right),\left(z_{1}, z_{2}\right)\right)
$$

and

$$
\begin{aligned}
& \max \left\{(\mu \times \lambda)\left(x_{1}, x_{2}\right),(\mu \lambda)\left(y_{1}, y_{2}\right),(\mu \times \lambda)\left(z_{1}, z_{2}\right)\right\} \\
& =\max \left\{\min \left\{\mu\left(x_{1}\right), \lambda\left(x_{2}\right)\right\}, \min \left\{\mu\left(y_{1}\right), \lambda\left(y_{2}\right)\right\}, \min \left\{\mu\left(z_{1}\right), \lambda\left(z_{2}\right)\right\}\right\} \\
& =\min \left\{\max \left\{\mu\left(x_{1}\right), \mu\left(y_{1}\right), \mu\left(z_{1}\right)\right\}, \max \left\{\lambda\left(x_{2}\right), \lambda\left(y_{2}\right), \lambda\left(z_{2}\right)\right\}\right\} \\
& \leq \min \left\{\max \left\{\mu\left(a_{1}\right), \mu\left(b_{1}\right), \mu\left(c_{1}\right)\right\}, \max \left\{\lambda\left(a_{2}\right), \lambda\left(b_{2}\right), \lambda\left(c_{2}\right)\right\}\right\} \\
& =\max \left\{\min \left\{\mu\left(a_{1}\right), \lambda\left(a_{2}\right)\right\}, \min \left\{\mu\left(b_{1}\right), \lambda\left(b_{2}\right)\right\}, \min \left\{\mu\left(c_{1}\right), \lambda\left(c_{2}\right)\right\}\right\} \\
& =\max \left\{(\mu \times \lambda)\left(a_{1}, a_{2}\right),(\mu \lambda)\left(b_{1}, b_{2}\right),(\mu \times \lambda)\left(c_{1}, c_{2}\right)\right\} .
\end{aligned}
$$

\section{References}

1. Biswas R (1990) Fuzzy subgroups and anti fuzzy subgroups. Fuzzy Sets \& Sys. 35, 121-124.

2. Davvaz B Fuzzy hyperideals in ternary semihyperrings. Iran J. Fuzzy Sys. 6(4), 21-36.

3. Davvaz B (1999) Fuzzy $H_{v}$-groups. Fuzzy Sets \& Sys. 101, 191-195.

4. Davvaz B and Leoreanu-Fotea V (2010) Intuitionistic fuzzy $n$-ary hypergroups. J. Multiple-Valued Logic \& Soft Comput. 16(1-2), 87-104.

5. Davvaz B and Corsini P (2007) Fuzzy $n$-ary
Vol. 5 No. 3 (Mar 2012)

ISSN: 0974- 6846 hypergroups. J. Intelligent \& Fuzzy Sys. 18(4), 377 382.

6. Davvaz B and Leoreanu-Fotea V (2010) Binary relations on ternary hypergroups. Commun. Algebra. 38(10), 3621-3636.

7. Davvaz B and Vougiouklis T (2006) n-Ary hypergroups. Iran. J. Sci. \& Technol. Trans. A30(A2), 165-174.

8. Davvaz B, Dehghan Nezad A and Benvidi A (2011) Chain reactions as experimental examples of ternary algebraic hyperstructures. MATCH Commun. Math. \& Comput. Chem. 65(2), 491-499.

9. Davvaz B, Kazanci O and Yamak S (2009) Intervalvalued fuzzy $n$-ary sub-hypergroups of $n$-ary hypergroups. Neural Comput. \& Appl. 18, 903-911.

10.Davvaz B, Corsini $\mathrm{P}$ and Leoreanu-Fotea V (2009) Atanassov's intuitionistic $(S, T)$-fuzzy $n$-ary subhypergroups and their properties. Inform. Sci. 179, 654-666.

11.Kazanc O, Davvaz B and Yamak S (2010) Fuzzy $n$ ary hypergroups related to fuzzy points. Neural Comput. \& Appl. 19, 649-655.

12.Kazanci O, Yamak S and Davvaz B (2011) On $n$-ary hypergroups and fuzzy $n$-ary homomorphism. Iran $\mathrm{J}$. Fuzzy Sys. 8(1), 1-17.

13.Rosenfeld A (1971) Fuzzy groups. J. Math. Anal. 35, 512-517.

14.Zadeh LA (1965) Fuzzy sets. Inform. \& Control. 8, 338-353.

15.Zhan J, Davvaz B and Shum KP (2010) Probabilistic $n$-ary hypergroups. Inform. Sci. 180, 1159-1166. 